\title{
The combined use of PD-1 antibodies and anti-angiogenic TKIs induces intractable thoracoabdominal wall ulcer in patients undergoing cryoablation for superficial metastases of hepatocellular carcinoma
}

\author{
shousheng Liu ( $\nabla$ liushsh@sysucc.org.cn ) \\ Sun Yat-sen University Cancer Center \\ Xun Wang \\ Sun Yat-sen University Cancer Center \\ Kunwei Peng \\ Guangzhou Medical University Second Affiliated Hospital \\ Xia Xu \\ Guangzhou Institute of Dermatology \\ Ning Lyu \\ Sun Yat-sen University Cancer Center \\ Meng $\mathrm{He}$ \\ Sun Yat-sen University Cancer Center \\ Bei Zhang \\ Sun Yat-sen University Cancer Center \\ Ming Zhao \\ Sun Yat-sen University Cancer Center
}

Research Article

Keywords: Hepatocellular carcinoma, cryoablation, PD-1 antibody, anti-angiogenic TKI.

Posted Date: February 21st, 2022

DOI: https://doi.org/10.21203/rs.3.rs-1173540/v1

License: () (7) This work is licensed under a Creative Commons Attribution 4.0 International License. Read Full License 


\section{Abstract}

Background: Percutaneous cryoablation has been widely used for local analgesia, especially for pain caused by superficial metastases. However, during the process of cryoablation for symptomatic superficial metastases of hepatocellular carcinoma (HCC), we observed that some patients developed intractable thoracoabdominal wall ulcer at the periphery of puncture points. In order to explore the potential causes of thoracoabdominal wall ulcer after cryoablation, we carried out this retrospective study.

Methods: We screened out 36 patients who underwent local cryoablation for superficial metastases (chest wall, abdominal wall, rib, clavicle and scapula) of HCC, of which 6 patients developed refractory thoracoabdominal wall ulcer. Clinical characteristics between ulcer and no ulcer groups were compared using the Pearson Chi square test. Risk factors for thoracoabdominal wall ulcer were screened using binary logistic regression model.

Results: All clinical factors, except for the administration of PD-1 antibodies plus anti-angiogenic tyrosine kinase inhibitors (TKIs) within 2 months before ( $p=$ 0.016) and after $(p=0.000)$ cryoablation, were balanced between the ulcer and no ulcer groups (all $p>0.05)$. Combined use of PD- 1 antibodies and antiangiogenic TKIs within 2 months after cryoablation was the only significant risk factor for thoracoabdominal wall ulcer (OR $=55.55,95 \%$ confidence interval (Cl) 2.62-1179.46, $\mathrm{p}=0.010$ ) in multivariate logistic regression model. Among the 8 patients who received combination therapy within 2 months after procedure, thoracoabdominal wall ulcer occurred in 5 patients (62.5\%). Among the 6 patients who developed thoracoabdominal wall ulcer, 3 patients died due to tumor progression, 2 patients improved their ulcers after PD-1 antibodies withdrawal and 1 patient was still under monitoring after stopping anti-PD-1 therapy.

Conclusions: HCC patients underwent combination therapy of PD-1 antibodies and anti-angiogenic TKls within 2 months after cryoablation for superficial metastases is prone to develop thoracoabdominal wall ulcer.

\section{Background}

Hepatocellular carcinoma (HCC) is the sixth most frequently diagnosed cancer globally, with more than 800 thousand new cases in 2018 , and the fourth largest cause of cancer-related death worldwide. It is estimated that approximate 1 million individuals will develop HCC each year by $2025^{1}$. Approximate 14.0 $36.7 \%$ of HCC patients have extrahepatic metastasis at initial diagnosis ${ }^{2}$, and the prognosis of patients with extrahepatic metastasis is poorer than that of those without it ${ }^{2}$. The frequent locations of extrahepatic metastasis are the lungs (45-55\%), regional and distant lymph nodes (53\%), bones (28-40\%), adrenal gland (12\%), peritoneum (11\%), and brain $(2 \%)^{2,4}$. In these patients with bone metastasis, the most common bone involvement sites are, in the order of descending frequency, the vertebrae, pelvis, ribs, skull and sternum ${ }^{5}$. Superficial metastases of HCC, except for rib metastasis, have also been reported in other locations such as chest wall, abdominal wall, scapula and clavicle ${ }^{6-9}$, although seldom seen.

Superficial metastases of HCC to bones, chest wall and abdominal wall often cause uncontrolled pain by irritating the periosteum, intercostal nerves, and the abdominal wall nerves ${ }^{10,11}$. In addition to systematic antitumor treatment, current treatment options for painful superficial metastases include systemic opioids, local radiotherapy and surgery. With long-term administration of opioids, patients' quality of life (QoL) was decreased due to side effects of nausea, constipation and sedation. Radiotherapy usually cannot be performed immediately and the response to radiotherapy may not be complete or rapid enough. With regard to surgery, substantial complications associated with chest wall resection such as delayed wound healing frequently occur ${ }^{12}$. Local non-invasive or minimally invasive interventions for pain palliation with few complications are needed to improve the QoL in these patients.

Recently, minimally invasive methods for pain palliation have emerged to deal with focal cancerous pain, including radiofrequency ablation (RFA), microwave ablation and cryoablation. These therapies possess the advantages of good patient compliance, rapid onset, high therapeutic efficacy, low incidence of complications, as well as local tumor control ${ }^{13}$. Among these methods, RFA has been most sufficiently evaluated with results from two multi-center researches demonstrating significantly alleviated focal pain in patients who are not sensitive to standard treatments ${ }^{14,15}$. With cryoablation, the ablation region is easily recognized as a low-density ice ball by CT imaging, and normal adjacent tissues are safe from frostbitten. In addition, with multi-probes composed, cryoablation can cover conformal margin of a larger tumor, and the ice ball holds the probe in place to prevent damage caused by any movement with breathing ${ }^{16}$. A further advantage of cryoablation is that patients underwent cryoablation would not experience exacerbated pain during the procedure or in the immediate post-operation period due to the analgesic effect of ice ${ }^{17}$. Therefore, cryoablation has been adopted as a preferred method for local analgesia, especially for pain caused by superficial metastases.

However, during the process of cryoablation for HCC superficial metastases to chest wall, abdominal wall, ribs, and so on, we observed that some patients developed refractory thoracoabdominal wall ulcer at the periphery of puncture points. In order to explore the potential causes of thoracoabdominal wall ulcer after cryoablation, we carried out this retrospective study.

\section{Methods}

\section{Patients}

Between January 2016 and June 2021, 321 patients diagnosed with primary HCC and underwent cryoablation treatment at Sun Yat-sen University Cancer Center were screened for eligibility in the present study. The inclusion criteria include: (1) diagnosed with advanced HCC; (2) superficial metastases of HCC, including thoracoabdominal wall, rib, clavicle and scapula; (3) received at least one cycle of cryoablation for superficial metastases; (4) and a more than 2 months follow-up period after cryoablation. Patients with one of the following circumstances were excluded: lack of critical systemic medication information; lack of chronic diseases information; and lack of ablation puncture point skin follow-up information. Among these patients, 285 patients were excluded for the 
following reasons: Cryoablation for non-superficial lesions ( $n=253)$, lack of systemic antitumor medication information ( $n=8)$, lack of anticoagulant or antiplatelet therapy information $(n=9)$, lack of information about diabetes mellitus $(n=5)$, and lack of skin ablation puncture point follow-up information ( $n=$ 10). The remaining 36 patients were finally included for following analysis. The screening process is summarized in Figure 1.

\section{Percutaneous cryoablation procedure}

The percutaneous cryoablation was performed using Visual-ICE ${ }^{T M}$ System (Galil Medical, Israel). Cryoablation treatments were performed by 3 senior interventional physicians (N.L, X.W and M.Z), each with at least 5 years of experience. Two to thirteen cryoprobes (diameter $1.47 \mathrm{~mm}$ ) were carefully percutaneously inserted into the tumor under CT guidance (Siemens Medical Solutions) after routine disinfection and local anesthesia. The number of probes was determined according to the tumor volume, geometry and location, with the aim of ensuring optimal tumor coverage. In case the estimated ice ball would reach the dermal layer of the skin, protective measures were adopted, such as placing warm saline on the skin and hydrodissection of subcutaneous tissues. When all probes and safeguards were prepared, procedure was initiated with adiabatic probes cooling to a temperature about subzero $160^{\circ} \mathrm{C}$ for $10-15$ min using argon gas, followed by rewarming to $30^{\circ} \mathrm{C}$ for $3 \mathrm{~min}$ with helium gas. Non-contrast CT scan was required every $3-5$ min intermittently to monitor the ice ball location and extent. Each cryoablation session consisted of two freezing-thawing cycles. Blood pressure, respiratory rate, pulse, and oxygen saturation were monitored during the procedure and for $12 \mathrm{~h}$ after ablation. Figure 2 shows the CT image of a representative patient during the cryoablation procedure. Anti-angiogenic tyrosine kinase inhibitors (TKIs) were discontinued two days before and after cryoablation.

\section{Monitoring and treatment of thoracoabdominal ulcer}

Six patients developed thoracoabdominal ulcer during post-ablation follow-up. Pathogen culture was performed on the ulcer surface of these patients to determine whether there was infection. For the ulcer of these patients, we adopted the following approach: clean up the superficial necrotic tissue, then fully disinfect the local area with non-irritating iodine, followed by applying silver ion dressing to promote healing. The above procedures were repeated every 3 days until the ulcer healed. If there was fluid leakage from the ulcer surface during the period, the dressing needed to be changed in time. For hyperkeratosis adjacent to the ulcer, keratin softeners such as urea ointment and salicylic acid vaseline were used topically. Besides, systemic treatment including nutritional support, immunity improvement and blood glucose control was performed concurrently.

\section{Statistical analysis}

Statistical analyses were conducted using SPSS 23.0 software (SPSS Inc, USA). Categorical characteristics between thoracoabdominal wall ulcer and no thoracoabdominal wall ulcer groups were compared using the Pearson Chi square test. The data of shortest ice ball-skin distance were presented as the mean \pm standard deviation, and independent sample t-test was conducted to compare the shortest ice ball-skin distance between the two groups. Binary logistic regression model was used to predict the odds ratio (OR) of risk factors for developing thoracoabdominal wall ulcer. Only variables with $\mathrm{p}$ value of less than 0.05 in the univariate model were introduced for subsequent multivariate analysis. A p value of less than 0.05 was considered statistically significant.

\section{Results}

\section{Patient characteristics}

A total of 36 metastatic HCC patients receiving superficial metastases (thoracoabdominal wall, rib, clavicle and scapula) cryoablation were brought into the present investigation, in which 6 patients developed thoracoabdominal ulcer after procedure. The baseline clinical characteristics of the 36 patients based on presence or absence of thoracoabdominal wall ulcer are shown in Table 1 . The great majority were male patients $(n=34,94.4 \%)$ and the median age was 55 years (range from 28 to 74 years). Twenty-six (72.2\%) patients had a liver function of Child-Pugh class A, and the remaining 10 (27.8\%) patients were in ChildPugh class B. Among all superficial metastases undergoing cryoablation, rib metastasis $(n=21,58.3 \%)$ was most common, followed by chest wall metastasis $(n=8,22.2 \%)$, scapula or clavicle metastasis $(n=4,11.1 \%)$, and the rarest is abdominal wall metastasis $(n=3,8.3 \%)$. None of the patients had infection at the ablation puncture point, and none received anticoagulant or antiplatelet drugs. A total of 10 patients underwent systemic administration of PD-1 antibodies plus anti-angiogenic TKIs after cryoablation, of which 8 patients were within 2 months and 6 patients also received combined treatment before cryoablation. Other 17 patients received single anti-angiogenic TKIs ( 6 apatinib, 8 sorafenib and 3 lenvatinib), 4 patients received anti-PD- 1 monotherapy ( 2 pembrolizumab and 2 nivolumab), and 5 patients received no systematic antitumor therapy within 2 months before and after cryoablation. 
Table 1

Baseline characteristics of HCC patients based on presence or absence of thoracoabdominal wall ulcer

\begin{tabular}{|c|c|c|c|}
\hline Characteristics & $\begin{array}{l}\text { No thoracoabdominal wall ulcer } \\
(\mathrm{N}=30)\end{array}$ & $\begin{array}{l}\text { Thoracoabdominal wall ulcer } \\
(\mathrm{N}=6)\end{array}$ & p-value \\
\hline \multicolumn{4}{|l|}{ Number of cases $(n, \%)$} \\
\hline Age (years) & & & 0.455 \\
\hline$<55$ & $15(50.0)$ & $2(33.3)$ & \\
\hline$\geq 55$ & $15(50.0)$ & $4(66.7)$ & \\
\hline Gender & & & 0.515 \\
\hline Male & 28 (93.3) & $6(100.0)$ & \\
\hline Female & $2(6.7)$ & $0(0.0)$ & \\
\hline WHO PS score & & & 0.371 \\
\hline $0-1$ & $16(53.3)$ & $2(33.3)$ & \\
\hline$\geq 2$ & $14(46.7)$ & $4(66.7)$ & \\
\hline Cryoablation tumor site & & & 0.142 \\
\hline Chest wall & $5(16.7)$ & $3(50.0)$ & \\
\hline Rib & $20(66.7)$ & $1(16.7)$ & \\
\hline Clavicle or scapula & $3(10.0)$ & $1(16.7)$ & \\
\hline Abdominal wall & $2(6.7)$ & $1(16.7)$ & \\
\hline Child-Pugh class & & & 0.506 \\
\hline A & $21(70.0)$ & $5(83.3)$ & \\
\hline B & $9(30.0)$ & $1(16.7)$ & \\
\hline Platelet $\left(\times 10^{9} / \mathrm{L}\right)$ & & & 0.829 \\
\hline$<100$ & $4(13.3)$ & $1(16.7)$ & \\
\hline$\geq 100$ & $26(86.7)$ & $5(83.4)$ & \\
\hline Hemoglobin (g/L) & & & 1.000 \\
\hline$<110$ & $5(16.7)$ & $1(16.7)$ & \\
\hline$\geq 110$ & 25 (83.3) & $5(83.4)$ & \\
\hline Albumin (g/L) & & & 0.131 \\
\hline$<35$ & $3(10.0)$ & $2(33.3)$ & \\
\hline$\geq 35$ & $27(90.0)$ & $4(66.7)$ & \\
\hline Total bilirubin $(\mu \mathrm{mol} / \mathrm{L})$ & & & 0.281 \\
\hline$<20$ & 25 (83.3) & $6(100.0)$ & \\
\hline$\geq 20$ & $5(16.7)$ & $0(0.0)$ & \\
\hline Prothrombin time (s) & & & 0.343 \\
\hline$<13.5$ & $26(86.7)$ & $6(100.0)$ & \\
\hline$\geq 13.5$ & $4(13.3)$ & $0(0.0)$ & \\
\hline Body mass index $\left(\mathrm{kg} / \mathrm{m}^{2}\right)$ & & & 0.418 \\
\hline$<18$ & $3(10.0)$ & $0(0.0)$ & \\
\hline$\geq 18$ & $27(90.0)$ & $6(100.0)$ & \\
\hline Diabetes mellitus & & & 0.418 \\
\hline Yes & $2(6.7)$ & $1(16.7)$ & \\
\hline No & $28(93.3)$ & $5(83.3)$ & \\
\hline Puncture point infection & & & - \\
\hline
\end{tabular}




\begin{tabular}{|c|c|c|c|}
\hline Characteristics & $\begin{array}{l}\text { No thoracoabdominal wall ulcer } \\
(\mathrm{N}=30)\end{array}$ & $\begin{array}{l}\text { Thoracoabdominal wall ulcer } \\
(\mathrm{N}=6)\end{array}$ & p-value \\
\hline Yes & $0(0.0)$ & $0(0.0)$ & \\
\hline No & $30(100.0)$ & $6(100.0)$ & \\
\hline Use of anticoagulant or antiplatelet agents & & & - \\
\hline Yes & $0(0.0)$ & $0(0.0)$ & \\
\hline No & $30(100.0)$ & $6(100.0)$ & \\
\hline Shortest ice ball-skin distance (mm) & $7.15 \pm 4.19$ & $5.67 \pm 1.72$ & 0.403 \\
\hline PD-1 plus TKI treatment within 2 months prior to cryoablation & & & 0.016 \\
\hline Yes & $3(10.0)$ & $3(50.0)$ & \\
\hline No & $27(90.0)$ & $3(50.0)$ & \\
\hline PD-1 plus TKI treatment within 2 months after cryoablation & & & 0.000 \\
\hline Yes & $3(10.0)$ & $5(83.3)$ & \\
\hline No & $27(90.0)$ & 1 (16.7) & \\
\hline
\end{tabular}

All clinical factors, including the factors that might affect wound healing, such as diabetes mellitus, body mass index, platelet, hemoglobin, albumin, prothrombin time and shortest ice ball-skin distance were evenly distributed between the two groups (all $p>0.05$ ), except for the administration of PD- 1 antibodies plus anti-angiogenic TKIs within 2 months before $(p=0.016)$ and after $(p=0.000)$ cryoablation. Among the 6 patients treated with combination therapy prior to cryoablation, 3 patients developed thoracoabdominal wall ulcer; while among the 8 patients who received combination therapy after procedure, thoracoabdominal wall ulcer occurred in 5 patients.

\section{Risk factor analysis for developing thoracoabdominal wall ulcer}

Binary logistic regression analysis was conducted to investigate the risk factors for developing thoracoabdominal wall ulcer after cryoablation. As shown in Table 2, systematic administration of PD-1 antibodies combined with anti-angiogenic TKIs within 2 months before and after cryoablation were risk factors for thoracoabdominal wall ulcer in univariate model (all $p<0.05$ ). However, when multivariate logistic regression was introduced, usage of PD-1 antibodies plus anti-angiogenic TKIs within 2 months after cryoablation was the only significant risk factor for thoracoabdominal wall ulcer $(\mathrm{OR}=55.55,95 \%$ confidence interval $(\mathrm{Cl}) 2.62-1179.46, \mathrm{p}=0.010)$. 
Table 2

Logistic regression analysis of risk factors for thoracoabdominal wall ulcer

\begin{tabular}{|c|c|c|c|c|}
\hline \multirow[t]{2}{*}{ Variables } & \multicolumn{2}{|l|}{ Univariate analysis } & \multicolumn{2}{|l|}{ Multivariate analysis } \\
\hline & OR $(95 \% \mathrm{Cl})$ & p-value & OR (95\% Cl) & p-value \\
\hline Age $(>55)$ & $2.00(0.32-12.62)$ & 0.461 & & \\
\hline Gender (Female) & - & - & & \\
\hline WHO PS score $(\geq 2)$ & $2.29(0.36-14.43)$ & 0.379 & & \\
\hline Cryoablation tumor site (reference, Chest wall) & - & 0.247 & & \\
\hline Child-Pugh class (B) & $0.47(0.05-4.58)$ & 0.513 & & \\
\hline Platelet $\left(<100 \times 10^{9} / \mathrm{L}\right)$ & $1.30(0.12-14.21)$ & 0.830 & & \\
\hline Hemoglobin $(<110 \mathrm{~g} / \mathrm{L})$ & $1.00(0.10-10.51)$ & 1.000 & & \\
\hline Albumin $(<35 \mathrm{~g} / \mathrm{L})$ & $4.50(0.57-35.83)$ & 0.155 & & \\
\hline Total bilirubin ( $\geq 20 \mu \mathrm{mol} / \mathrm{L})$ & - & - & & \\
\hline Prothrombin time ( $\geq 13.5 \mathrm{~s})$ & - & - & & \\
\hline Body mass index $(<18 \mathrm{~kg} / 囚)$ & - & - & & \\
\hline Diabetes mellitus (Yes) & $2.80(0.21-37.03)$ & 0.434 & & \\
\hline Puncture point infection (Yes) & - & - & & \\
\hline Use of anticoagulant or antiplatelet agents (Yes) & - & - & & \\
\hline PD-1 plus TKI treatment within 2 months prior to cryoablation (Yes) & $9.00(1.22-66.23)$ & 0.031 & $0.71(0.04-13.12)$ & 0.815 \\
\hline PD-1 plus TKI treatment within 2 months after cryoablation (Yes) & $45.00(3.86-524.68)$ & 0.002 & $55.55(2.62-1179.46)$ & 0.010 \\
\hline OR: odds ratio; 95\% Cl: 95\% Confidence interval & & & & \\
\hline
\end{tabular}

\section{Details of the 6 patients developing thoracoabdominal ulcer}

Details of the patients developed thoracoabdominal ulcer are summarized in Table3. These patients were followed up for 2 to 42 months (mean: 12 months). A total of 6 male patients aged between 42-74 years developed painless ulcer, including 5 chest wall ulcer and 1 abdominal wall ulcer. Of the 5 patients with chest wall ulcer, 3 underwent cryoablation on chest wall metastases, and the other 2 underwent cryoablation on scapular and rib metastases respectively. The mean value of shortest ice ball-skin distance was $5.67 \pm 1.72 \mathrm{~mm}$, which had no significant difference compared with the other 30 patients without thoracoabdominal ulcer $(7.15 \pm 4.19 \mathrm{~mm}, \mathrm{p}=0.403)$. Before cryoablation, 2 patients received no systematic treatment, 1 patient received single-agent sorafenib for 4 months, and the remaining 3 patients received 1-32 cycles of PD-1 antibodies plus anti-angiogenic TKIs treatment with the last administration within 2 months before cryoablation. In terms of post-ablation treatment, 1 patient continued to take single-agent sorafenib for 302 days until the disease progressed. The remaining 5 patients received 1-16 cycles of combination therapy with the first administration within 2 months after cryoablation (14-42 days), in which anti-angiogenic TKls were administrated continuously until the disease progressed. Based on our later understanding that combination therapy may induce and worsen thoracoabdominal ulcer, some patients discontinued PD-1 antibodies, leaving anti-angiogenic TKIs alone after sufficiently balancing tumor control and ulcer. 
Table 3

Clinical features of 6 patients with thoracoabdominal wall ulcer

\begin{tabular}{|c|c|c|c|c|c|c|c|c|c|c|}
\hline Patient & Gender & $\begin{array}{l}\text { Age } \\
\text { (years) }\end{array}$ & $\begin{array}{l}\text { Cryoablation } \\
\text { tumor site }\end{array}$ & $\begin{array}{l}\text { Shortest } \\
\text { ice ball- } \\
\text { skin } \\
\text { distance } \\
(\mathrm{mm})\end{array}$ & $\begin{array}{l}\text { Medication } \\
\text { prior to } \\
\text { cryoablation }\end{array}$ & $\begin{array}{l}\text { Preoperative } \\
\text { medication } \\
\text { cycles }\end{array}$ & $\begin{array}{l}\text { Time from } \\
\text { last } \\
\text { preoperative } \\
\text { medication } \\
\text { to } \\
\text { cryoablation }\end{array}$ & $\begin{array}{l}\text { Medication } \\
\text { after } \\
\text { cryoablation }\end{array}$ & $\begin{array}{l}\text { Postoperative } \\
\text { medication } \\
\text { cycles }\end{array}$ & $\begin{array}{l}\text { Time frol } \\
\text { cryoabla1 } \\
\text { to first } \\
\text { postoper } \\
\text { medicati }\end{array}$ \\
\hline \multirow[t]{2}{*}{1} & Male & 63 & $\begin{array}{l}\text { Abdominal } \\
\text { wall }\end{array}$ & 8.50 & $\begin{array}{l}\text { Pembrolizumab } \\
200 \mathrm{mg} \text { q3w + } \\
\text { Lenvatinib }\end{array}$ & 2 cycles & 6 days & $\begin{array}{l}\text { Pembrolizumab } \\
200 \mathrm{mg} \text { q3w + } \\
\text { Lenvatinib }\end{array}$ & 6 cycles & 15 days \\
\hline & & & & & $8 \mathrm{mg} \mathrm{qd}$ & & & $8 \mathrm{mg} \mathrm{qd}$ & & \\
\hline \multirow[t]{2}{*}{2} & Male & 66 & Scapula & 6.40 & $\begin{array}{l}\text { Camrelizumab } \\
200 m g \text { q3w + } \\
\text { Lenvatinib }\end{array}$ & 1 cycle & 44 days & $\begin{array}{l}\text { Camrelizumab } \\
\text { 200mg q3w + } \\
\text { Lenvatinib }\end{array}$ & 1 cycle & 35 days \\
\hline & & & & & $8 \mathrm{mg} \mathrm{qd}$ & & & $8 \mathrm{mg}$ qd & & \\
\hline \multirow[t]{2}{*}{3} & Male & 42 & Chest wall & 3.30 & Non & Non & Non & $\begin{array}{l}\text { Camrelizumab } \\
200 m g \text { q3w + } \\
\text { Sorafenib }\end{array}$ & 10 cycles & 26 days \\
\hline & & & & & & & & $0.8 \mathrm{~g} \mathrm{qd}$ & & \\
\hline \multirow[t]{2}{*}{4} & Male & 50 & Chest wall & 5.60 & $\begin{array}{l}\text { Nivolumab } \\
240 m g \text { q2w + } \\
\text { Lenvatinib }\end{array}$ & 32 cycles & 7 days & $\begin{array}{l}\text { Nivolumab } \\
240 \mathrm{mg} \text { q2w + } \\
\text { Lenvatinib }\end{array}$ & 16 cycles & 14 days \\
\hline & & & & & $8 \mathrm{mg} q d$ & & & $8 \mathrm{mg} q d$ & & \\
\hline \multirow[t]{2}{*}{5} & Male & 69 & Chest wall & 5.20 & Non & Non & Non & $\begin{array}{l}\text { Camrelizumab } \\
200 \mathrm{mg} \text { q3w + } \\
\text { Apatinib }\end{array}$ & 2 cycles & 42 days \\
\hline & & & & & & & & $0.5 \mathrm{~g} \mathrm{qd}$ & & \\
\hline \multirow[t]{2}{*}{6} & Male & 74 & Rib & 5.00 & Sorafenib & 122 days & 2 days & Sorafenib & 302 days & 2 days \\
\hline & & & & & $0.8 \mathrm{~g} \mathrm{qd}$ & & & $0.8 \mathrm{~g} \mathrm{qd}$ & & \\
\hline
\end{tabular}


nearly through the whole thoracoabdominal wall, and the surface was covered with necrotic tissue. No pathogen or tumor invasion was observed on the ulcer surface.

In 2 patients, after discontinuing PD-1 antibodies, the necrotic tissue on the ulcer surface fell off by degrees, followed by fresh granulation tissue forming from the surrounding and base of the ulcer. The ulcer area gradually decreased and the depth gradually became shallow. Up to the last follow-up, the ulcers were still healing progressively. Three patients, including 1 patient who received single-agent sorafenib after cryoablation, died due to tumor progression with no improvement in ulcers. Another patient was instructed to cease anti-PD-1 therapy 2 weeks before the last follow-up, and the ulcer was still under monitoring. The details and ulcer changes of each patient are shown in Table 3. Typical features and dynamic changes of thoracoabdominal ulcer are shown in Figure 3. It should be noted that the patients in the control group also developed frostbite on the local skin after cryoablation, but the degree of skin damage was mild, and most of them were limited in epidermal layers. Generally, the lesions could be cured within 2-3 weeks after local treatment. In addition, we also found that thoracoabdominal wall ulcer did not emerge in 3 of the 8 patients who received combination treatment within 2 months after cryoablation. Their clinical characteristics are shown in Table S1 as supplementary materials. Two patients were treated with sintilimab plus anti-angiogenic TKIs, and 1 patient was treated with toripalimab plus anti-angiogenic TKI.

\section{Discussion}

We performed the present study in order to explore the potential causes of thoracoabdominal wall ulcer after cryoablation, and found that the combined use of PD-1 antibodies and anti-angiogenic TKIs within 2 months after cryoablation for superficial tumor is the most important risk factor for local refractory ulcer at puncture point.

Systemic therapies are recommended for HCC patients with advanced disease (BCLC-C stage). Sorafenib remained the unique effective option for first-line therapy until lenvatinib, another multiple kinase inhibitor, showed favorable clinical outcomes in a non-inferiority trial. Median overall survival was 12.3 months with sorafenib and 13.6 months with lenvatinib ${ }^{18,19}$. In recent years, significant progress has been made in exploring new and efficient systemic therapies for advanced HCC. Based on phase Ib/II clinical trial data, pembrolizumab and nivolumab (anti-PD-1 antibodies) were also approved as single agents for alternative treatment of advanced HCC in second-line settings ${ }^{20,21}$. Other common anti-angiogenic TKIs or PD-1 antibodies, such as apatinib, camrelizumab, toripalimab and sintilimab, have also been proved to be effective in advanced HCC and applied clinically ${ }^{22-24}$. Although anti-angiogenic or anti-PD-1 monotherapy can achieve certain therapeutic effects, new combination strategies are being developed to enhance tumor responsiveness to PD-1 inhibition across tumor types in oncology 25 . The vascular endothelial growth factor (VEGF) signaling pathway promotes localized immunosuppression by inhibiting antigen-presenting cells and effector $C D 8^{+} T$ cells as well as by activating immune suppressor cells such as Treg cells and myeloid- derived suppressor cells, providing a theoretical basis for combining immune checkpoint inhibitors with anti-angiogenic agents ${ }^{26}$. A phase lb clinical trial of pembrolizumab plus lenvatinib as frontline treatment in 100 unresectable HCC patients produced objective and durable radiographic responses in $46 \%$ of participants, with a median progression free survival of 9.5 months and a median overall survival of 22 months ${ }^{27}$. Several other studies have also demonstrated that PD-1 antibodies synergized with anti-angiogenic TKIs in advanced HCC ${ }^{28,29}$. More important, based on IMbrave150 trial, atezolizumab (anti-PD-L1 antibody) plus bevacizumab has become the standard first-line therapy for advanced $\mathrm{HCC}^{30}$. Therefore, the combination therapy strategy is becoming increasingly adopted in clinic to further improve the curative effect.

Image-guided ablations, including percutaneous RFA, microwave ablation, cryoablation and so on, are recommended as a curative therapy for small, earlystage (BCLC 0 or A stage) HCC tumors ${ }^{31}$. Beyond the standard indications, ablation is also accepted as an alternative method for palliative and analgesic treatment of metastatic $\mathrm{HCC}$, especially for the superficial metastases to bones and thoracoabdominal wall that cause intractable pain, in which condition cryoablation is most commonly used due to its unique advantages in pain relief ${ }^{17}$. In this case, cryoablation is often employed in conjunction with systemic therapy including the combined use of PD-1 antibodies and anti-angiogenic TKIs.

Bevacizumab, a VEGF inhibitor, can bring about wound healing complications via preventing vascular reconstruction of damaged tissues, resulting in interruption of the proliferation and granulation stages in healing process ${ }^{32}$. Surgical wound healing complications related to bevacizumab have been reported[33]. Multiple kinase inhibitors such as lenvatinib, sorafenib, apatinib and regorafenib can also inhibit VEGF/VEGFR, thereby affecting angiogenesis. Whether these anti-angiogenic TKIs, especially when in combination with PD-1 antibodies will affect the local wound healing after invasive procedure such as percutaneous ablation is rarely reported.

A total of 36 patients were included in the study, all of whom obtained effective local tumor control pain relief after cryoablation. Most of the patients received anti-angiogenic TKIs or anti-PD-1 therapy during perioperative period, and 6 of them developed thoracoabdominal ulcer. All the TKIs used in the enrolled individuals including lenvatinib, sorafenib, apatinib and regorafenib can inhibit VEGFR, especially VEGFR2, which is a major signal transducer in angiogenesis ${ }^{34}$. Inhibition of angiogenesis results in cell death due to tissue hypoxia, and eventually caused delayed wound healing complications ${ }^{35}$. However, unlike bevacizumab, there are few reports on delayed wound healing caused by single-agent anti-angiogenic TKIs in clinical practice. In a series of case report, 11 patients with advanced thyroid cancer being treated with lenvatinib underwent invasive procedures including central venous catheter implantation, cataract surgery, percutaneous coronary angioplasty, percutaneous endoscopic gastrostomy, and thoracic catheterization, and delayed wound healing was observed in only one patient ${ }^{36}$. The mechanism of the different influence on wound healing between bevacizumab and lenvatinib remains unclear, which may be due to their remarkable discrepancy in half-life. The half-life of bevacizumab is 21 days, while that of lenvatinib is only 28 hours and similar to other anti-angiogenic TKIs ${ }^{37,38}$, in which situation the wound is readily to reverse through drug discontinuation. Similar to previous report, based on our data, of the 17 patients who received single-agent TKls before and after cryoablation, only 1 patient with sorafenib developed thoracoabdominal ulcer, with the incidence of only $5.88 \%$. However, for patients who received the combined therapy within 2 months after cryoablation, the incidence of thoracoabdominal wall ulcer was as high as $62.5 \%$ (5 of 8). Cryoablation often causes skin damage when the ice ball extends to the vicinity of skin. The shortest ice ball-skin 
distance is the most important risk factor for skin damage ${ }^{39}$. In our study, some protective measures such as placing warm saline on the skin and hydrodissection of subcutaneous tissues were taken, and most of the frostbite on local skin after cryoablation was mild and could be cured within 2-3 weeks through supportive care. In addition, there was no significant difference in shortest ice ball-skin distance between the ulcer group and the no ulcer group. Therefore, we speculate that the formation of thoracoabdominal ulcer is not, or at least not mainly, caused by the cryoablation procedure itself. Other factors that may affect wound healing, such as age, nutritional status, diabetes, serum albumin level, were all balanced between two groups. Besides, no patient took anticoagulant or antiplatelet agents, and no patient was complicated with puncture point infection. Based on the above evidences, we demonstrated that, among all the potential risk factors, combined use of PD-1 antibodies and anti-angiogenic TKIs within 2 months after cryoablation was the main cause of thoracoabdominal wall ulcer.

The mechanism by which combination therapy, rather than single-agent anti-angiogenic TKIs, can significantly increase the risk of thoracoabdominal ulcer remains unclear. The administration of TKIs that inhibit VEGFR leads to an increase in dendritic cell antigen presentation, promotes T cell activation in the start-up phase, and facilitates the migration of T cells to the tumor site by means of normalizing the tumor blood vessels ${ }^{40,41}$. Besides, anti-VEGFR TKIs also inhibit the activity of immune suppressor cells such as Treg cells and myeloid-derived suppressor cells, leading to the transformation of the immunosuppressive microenvironment to an immune-activated microenvironment[42]. It has been reported that CD $4^{+} \mathrm{T}$ lymphocytes promote healing by positively affecting the wound micro-environment, while $C D 8^{+} T$ lymphocytes impede healing by creating a cytokine environment that antagonizes $C D 4^{+} T$ lymphocyte-mediated process ${ }^{43}$. Treg cells also play an important role in wound healing, both by inhibiting local inflammation and by directly promoting the tissue regeneration process through constitutive release of bioactive TGF- $\beta$ within tissues ${ }^{44}$. The administration of anti-PD- 1 agents under such conditions further enhances the activity of $C D 8^{+} \mathrm{T}$ cells as well as inhibits the function of Treg cells, which aggravates the local inflammatory reaction of the wound, resulting in delayed wound healing and the formation of ulcer.

In addition to cryoablation, other invasive procedures, such as surgery, when performed during the combined use of PD- 1 antibodies and anti-angiogenic TKIs, may also lead to delayed wound healing or ulcer, which would seriously affect the QoL of these patients and needs to be paid enough attention. Moreover, superficial ulcer is visible, but deep tissue changes are difficult to monitor. Delayed healing of the viscera is often complicated with occult bleeding and infection, sometimes fatal. Therefore, we need to fully weigh the advantages and disadvantages for these patients who receive invasive procedures during combination therapy and closely monitor them after procedures.

Several deficiencies existed in our research. First, only 6 patients developed thoracoabdominal wall ulcer, a sample size too small to draw convincing conclusions. However, it is precisely because we observed that the combined treatment group is more prone to develop thoracoabdominal ulcer after cryoablation, we ceased cryoablation on patients receiving combined treatment, so as to avoid more patients from thoracoabdominal ulcer. In this case, expanding the sample size is unethical. In addition, we also terminated PD-1 antibodies administration in patients with ulcer after realizing this problem, and the ulcer of some patients could be improved. Second, we failed to conducted pathological biopsy in the area of the ulcer to exclude the ulcer caused by tumor invasion and to understand local lymphocyte infiltration, because invasive biopsy on the ulcer surface is unfavorable to wound repair, and we had been observing the ulcer and found no local tumor growth. Furthermore, the mechanism by which the combination therapy significantly increases the risk of thoracoabdominal ulcer remains unclear, which needs to be investigated by basic research in the future.

\section{Conclusion}

In conclusion, based on our study, the combined use of PD-1 antibodies and anti-angiogenic TKls within 2 months after cryoablation for superficial tumor causes local refractory ulcer at puncture point. If cryoablation is needed for relieving the pain caused by superficial metastases, combined therapy should be transformed into monotherapy within 2 months after cryoablation, or other symptomatic treatment methods such as drug analgesia should be adopted to replace cryoablation.

\section{Abbreviations}

HCC, hepatocellular carcinoma; TKIs, angiogenic tyrosine kinase inhibitors; QoL, quality of life; RFA, radiofrequency ablation.

\section{Declarations}

Ethics approval and consent to participate: The study was conducted in accordance with the principles of the Declaration of Helsinki and was authorized by the Research Ethics Committee of Sun Yat-sen University. Written informed consent was obtained from individual or guardian participants.

Consent for publication: Written informed consent for publication was obtained from study participants.

Availability of data and materials: The raw data of this article has been uploaded onto the Research Data Deposit public platform (www.researchdata.org.cn). The datasets used and/or analyzed during the current study are also available from the corresponding author on reasonable request.

Competing interests: The authors declare that they have no conflicts of interest.

Funding: This research was funded by National Natural Science Foundation of China (82102995 to Shousheng Liu). 
Authors' contributions: S-SL, XW, BZ and MZ designed and performed the research; K-WP, NL and MH collected and analyzed the data; XX was responsible for guiding ulcer care and the follow-up of ulcer status; S-SL and XW analyzed the results and wrote the manuscript. All authors read and approved the final manuscript.

Acknowledgments: The authors thank all the staff in the follow-up room from Sun Yat-sen University Cancer Center for their help in the present study.

\section{References}

1. Llovet JM, Kelley RK, Villanueva A, et al. Hepatocellular carcinoma. NAT REV DIS PRIMERS. 2021;7(1):6

2. Katyal S, Oliver JR, Peterson MS, Ferris JV, Carr BS, Baron RL. Extrahepatic metastases of hepatocellular carcinoma. RADIOLOGY. 2000;216(3):698-703.

3. Elmoghazy W, Ahmed K, Vijay A, et al. Hepatocellular carcinoma in a rapidly growing community: Epidemiology, clinico-pathology and predictors of extrahepatic metastasis. ARAB J GASTROENTEROL. 2019;20(1):38-43.

4. Yi J, Gwak GY, Sinn DH, et al. Screening for extrahepatic metastases by additional staging modalities is required for hepatocellular carcinoma patients beyond modified UICC stage T1. Hepatogastroenterology. 2013;60(122):328-32.

5. Ruiz-Morales JM, Dorantes-Heredia R, Chable-Montero F, Vazquez-Manjarrez S, Mendez-Sanchez N, Motola-Kuba D. Bone metastases as the initial presentation of hepatocellular carcinoma. Two case reports and a literature review. ANN HEPATOL. 2014;13(6):838-42.

6. Daga D, Dana R, Kothari N. Hepatocellular carcinoma - an unusual metastatic presentation on the chest wall. Clin Exp Hepatol. 2016;1(4):133-5.

7. Goto M, Sakamoto K, Kawano S, et al. A case of simultaneous abdominal wall metastasis of hepatocellular carcinoma with long-term relapse-free survival after laparoscopic resection. Int Cancer Conf J. 2021;10(3):217-21.

8. Lee LC, Tsuei YC. Metastasis of hepatocellular carcinoma to the scapula. J FORMOS MED ASSOC. 2014;113(12):976-7.

9. Cebrian A, Elosua A, Gonzalez-de LHB, Irisarri R, Ruiz-Clavijo D. Clavicle tumor as an initial manifestation of fibrolamellar hepatocellular carcinoma. Rev Gastroenterol Mex (Engl Ed). 2020;85(1):104-6.

10. MacLeod N, Kelly C, Stobo J, et al. Pain in Malignant Pleural Mesothelioma: A Prospective Characterization Study. PAIN MED. 2016;17(11):2119-26.

11. Urch C. The pathophysiology of cancer-induced bone pain: current understanding. Palliat Med. 2004;18(4):267-74.

12. Lans TE, van der Pol C, Wouters MW, Schmitz PI, van Geel AN. Complications in wound healing after chest wall resection in cancer patients; a multivariate analysis of 220 patients. J THORAC ONCOL. 2009;4(5):639-43.

13. Arrigoni F, Bruno F, Zugaro L, et al. Developments in the management of bone metastases with interventional radiology. Acta Biomed. 2018;89(1-S):16674.

14. Goetz MP, Callstrom MR, Charboneau JW, et al. Percutaneous image-guided radiofrequency ablation of painful metastases involving bone: a multicenter study. J CLIN ONCOL. 2004;22(2):300-6.

15. Dupuy DE, Liu D, Hartfeil D, et al. Percutaneous radiofrequency ablation of painful osseous metastases: a multicenter American College of Radiology Imaging Network trial. CANCER-AM CANCER SOC. 2010;116(4):989-97.

16. Santiago E, Pauly V, Brun G, Guenoun D, Champsaur P, Le Corroller T. Percutaneous cryoablation for the treatment of osteoid osteoma in the adult population. EUR RADIOL. 2018;28(6):2336-44.

17. Thacker PG, Callstrom MR, Curry TB, et al. Palliation of painful metastatic disease involving bone with imaging-guided treatment: comparison of patients' immediate response to radiofrequency ablation and cryoablation. AJR Am J Roentgenol. 2011;197(2):510-5.

18. Llovet JM, Ricci S, Mazzaferro V, et al. Sorafenib in advanced hepatocellular carcinoma. N Engl J Med. 2008;359(4):378-90.

19. Kudo M, Finn RS, Qin S, et al. Lenvatinib versus sorafenib in first-line treatment of patients with unresectable hepatocellular carcinoma: a randomised phase 3 non-inferiority trial. LANCET. 2018;391(10126):1163-73.

20. Yau T, Kang YK, Kim TY, et al. Efficacy and Safety of Nivolumab Plus Ipilimumab in Patients With Advanced Hepatocellular Carcinoma Previously Treated With Sorafenib: The CheckMate 040 Randomized Clinical Trial. JAMA ONCOL. 2020;6(11):e204564.

21. Zhu AX, Finn RS, Edeline J, et al. Pembrolizumab in patients with advanced hepatocellular carcinoma previously treated with sorafenib (KEYNOTE-224): a non-randomised, open-label phase 2 trial. LANCET ONCOL. 2018;19(7):940-52.

22. Qin S, Li Q, Gu S, et al. Apatinib as second-line or later therapy in patients with advanced hepatocellular carcinoma (AHELP): a multicentre, double-blind, randomised, placebo-controlled, phase 3 trial. Lancet Gastroenterol Hepatol. 2021;6(7):559-68.

23. Qin S, Ren Z, Meng Z, et al. Camrelizumab in patients with previously treated advanced hepatocellular carcinoma: a multicentre, open-label, parallel-group, randomised, phase 2 trial. LANCET ONCOL. 2020;21(4):571-80.

24. Chen J, Hu X, Li Q, et al. Effectiveness and safety of toripalimab, camrelizumab, and sintilimab in a real-world cohort of hepatitis B virus associated hepatocellular carcinoma patients. Ann Transl Med. 2020;8(18):1187.

25. Zappasodi R, Merghoub T, Wolchok JD. Emerging Concepts for Immune Checkpoint Blockade-Based Combination Therapies. CANCER CELL. 2018;33(4):581-98.

26. Rahma OE, Hodi FS. The Intersection between Tumor Angiogenesis and Immune Suppression. CLIN CANCER RES. 2019;25(18):5449-57.

27. Finn RS, Ikeda M, Zhu AX, et al. Phase Ib Study of Lenvatinib Plus Pembrolizumab in Patients With Unresectable Hepatocellular Carcinoma. J CLIN ONCOL. 2020;38(26):2960-70.

28. Xu J, Shen J, Gu S, et al. Camrelizumab in Combination with Apatinib in Patients with Advanced Hepatocellular Carcinoma (RESCUE): A Nonrandomized, Open-label, Phase II Trial. CLIN CANCER RES. 2021;27(4):1003-11. 
29. Zheng L, Fang S, Wu F, et al. Efficacy and Safety of TACE Combined With Sorafenib Plus Immune Checkpoint Inhibitors for the Treatment of Intermediate and Advanced TACE-Refractory Hepatocellular Carcinoma: A Retrospective Study. Front Mol Biosci. 2020; 7(609322.

30. Finn RS, Qin S, Ikeda M, et al. Atezolizumab plus Bevacizumab in Unresectable Hepatocellular Carcinoma. N Engl J Med. 2020;382(20):1894-905.

31. Heimbach JK, Kulik LM, Finn RS, et al. AASLD guidelines for the treatment of hepatocellular carcinoma. HEPATOLOGY. 2018;67(1):358-80.

32. Bates DO, Jones RO. The role of vascular endothelial growth factor in wound healing. Int J Low Extrem Wounds. 2003;2(2):107-20.

33. Scappaticci FA, Fehrenbacher L, Cartwright T, et al. Surgical wound healing complications in metastatic colorectal cancer patients treated with bevacizumab. J SURG ONCOL. 2005;91(3):173-80.

34. Shibuya M. Vascular Endothelial Growth Factor (VEGF) and Its Receptor (VEGFR) Signaling in Angiogenesis: A Crucial Target for Anti- and Pro-Angiogenic Therapies. Genes Cancer. 2011;2(12):1097-105.

35. Moslehi JJ. Cardiovascular Toxic Effects of Targeted Cancer Therapies. N Engl J Med. 2016;375(15):1457-67.

36. Toda S, Iwasaki H, Murayama D, Nakayama H, Suganuma N, Masudo K. Invasive procedures in patients undergoing treatment with lenvatinib for thyroid cancer. Mol Clin Oncol. 2021;14(4):81.

37. Gordon MS, Margolin K, Talpaz M, et al. Phase I safety and pharmacokinetic study of recombinant human anti-vascular endothelial growth factor in patients with advanced cancer. J CLIN ONCOL. 2001;19(3):843-50.

38. Yamada K, Yamamoto N, Yamada Y, et al. Phase I dose-escalation study and biomarker analysis of E7080 in patients with advanced solid tumors. CLIN CANCER RES. 2011;17(8):2528-37.

39. Maybody M, Tang PQ, Moskowitz CS, Hsu M, Yarmohammadi H, Boas FE. Pneumodissection for skin protection in image-guided cryoablation of superficial musculoskeletal tumours. EUR RADIOL. 2017;27(3):1202-10.

40. Gabrilovich DI, Chen HL, Girgis KR, et al. Production of vascular endothelial growth factor by human tumors inhibits the functional maturation of dendritic cells. NAT MED. 1996;2(10):1096-103.

41. Goel S, Duda DG, Xu L, et al. Normalization of the vasculature for treatment of cancer and other diseases. PHYSIOL REV. 2011;91(3):1071-121.

42. Hegde PS, Wallin JJ, Mancao C. Predictive markers of anti-VEGF and emerging role of angiogenesis inhibitors as immunotherapeutics. SEMIN CANCER BIOL. 2018;52(Pt 2):117-24.

43. Keen D. A review of research examining the regulatory role of lymphocytes in normal wound healing. J WOUND CARE. 2008;17(5):218-20, 222.

44. Zaiss DM, Minutti CM, Knipper JA. Immune- and non-immune-mediated roles of regulatory T-cells during wound healing. IMMUNOLOGY. 2019;157(3):190-7.

\section{Figures}


HCC patients treated with

cryoablation between January 2016

and June $2021(\mathrm{n}=321)$

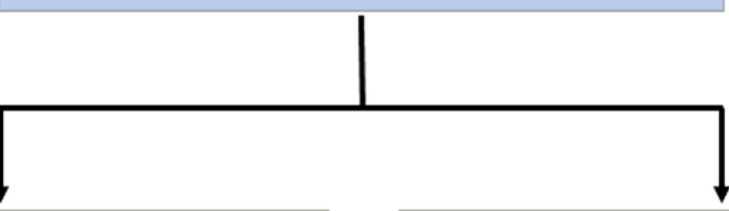

Cryoablation on superficial

metastases, including thoracoabdominal wall, rib, clavicle and scapula $(\mathrm{n}=68)$

Cryoablation non-superficial lesions $(\mathrm{n}=253)$

$$
\mid \begin{aligned}
& \text { Excluded: } \\
& \text { Lack of systemic antitumor medication } \\
& \text { information }(\mathrm{n}=8) \\
& \text { Lack of anticoagulant or antiplatelet therapy } \\
& \text { information }(\mathrm{n}=9) \\
& \text { Lack of information about diabetes mellitus }(\mathrm{n}=5) \\
& \text { Lack of skin ablation puncture point follow-up } \\
& \text { information }(\mathrm{n}=10)
\end{aligned}
$$

Included for study

$(n=36)$

Figure 1

Flow chart depicting patient deposition. HCC, hepatocellular carcinoma

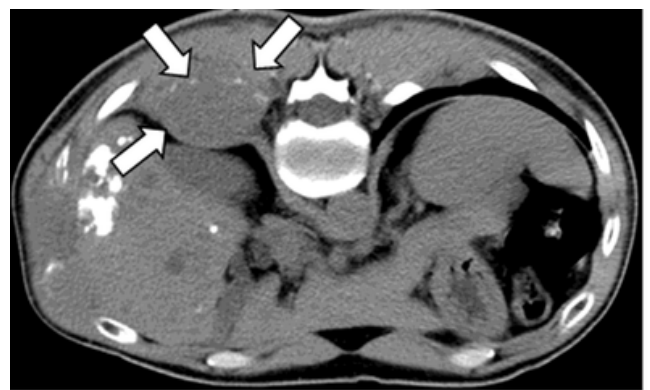

(A)

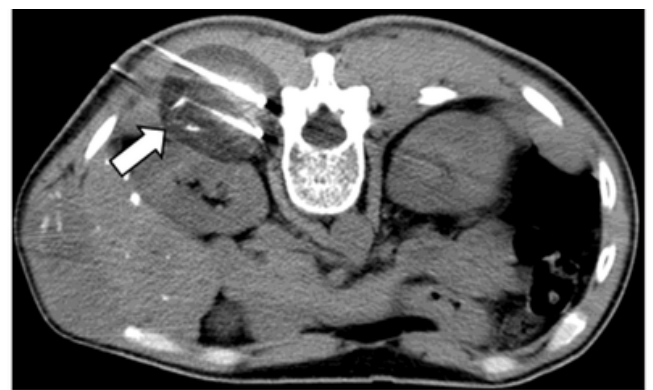

(B)

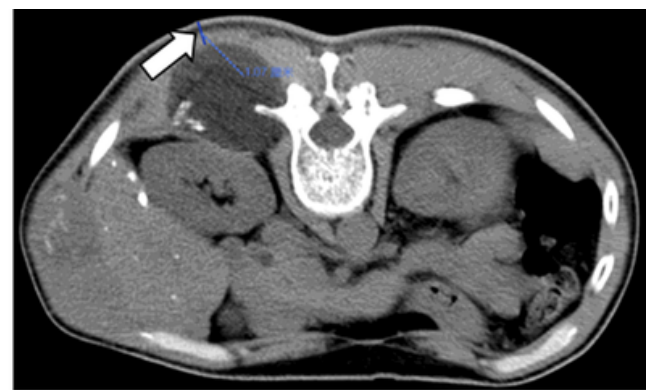

(C)

Figure 2

Representative CT imaging during the cryoablation of a metastasis in the rib. (A) Non-contrast CT scan of the rib metastasis before cryoablation showed a round low-density soft tissue shadow on the right rib (arrows); (B) Non-contrast CT imaging shows the ice ball as a low attenuation area around the cryoprobes, exceeding beyond the margin of tumor (arrow). Two cryoprobes are placed in the metastasis; (C) Shortest ice ball-skin distance, as shown in the blue line (arrow). 


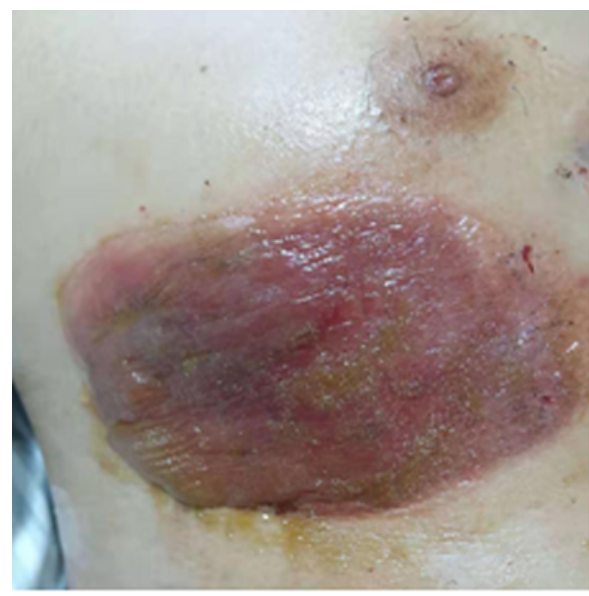

(A)

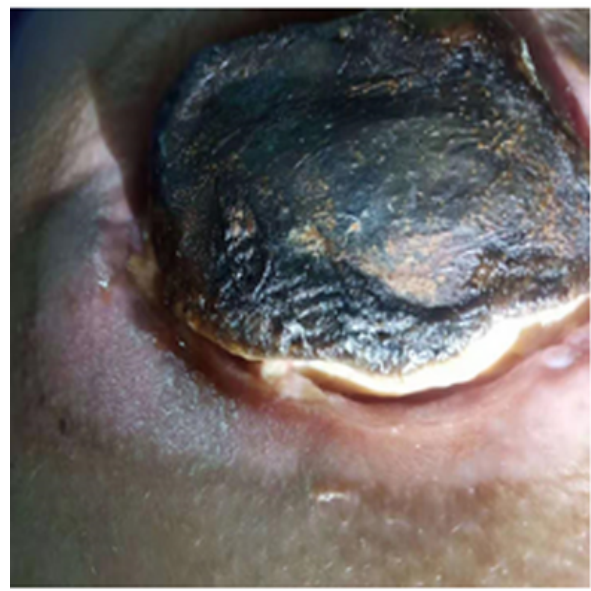

(D)

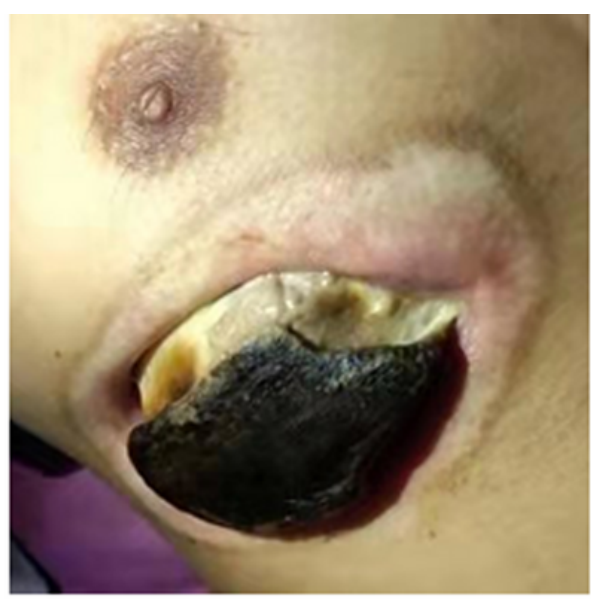

(B)

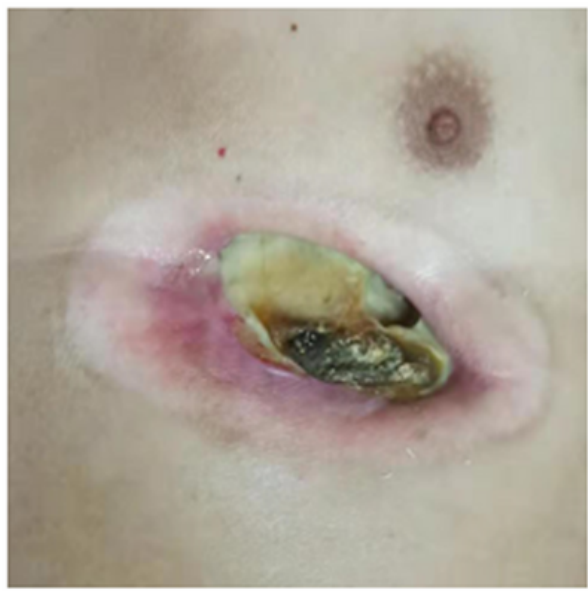

(E)

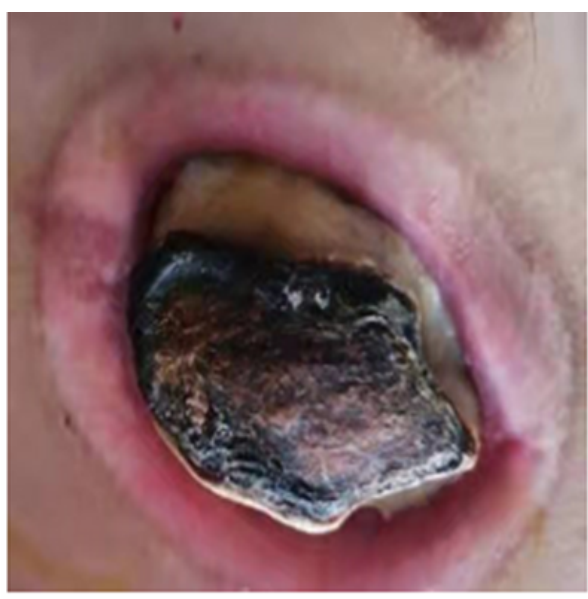

(C)

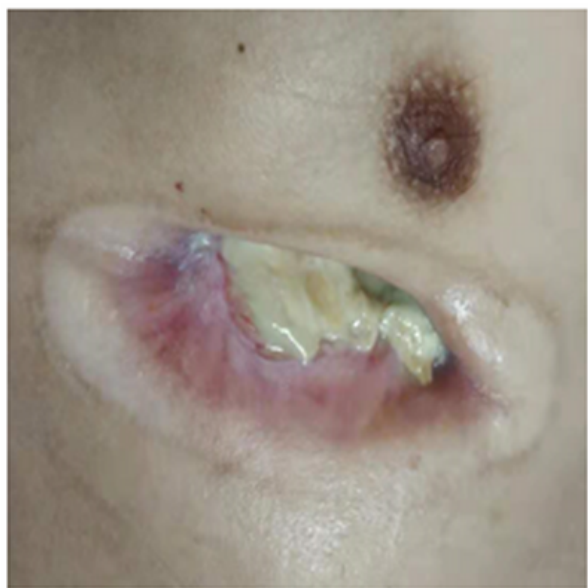

(F)

\section{Figure 3}

Representative characteristics and dynamic changes of thoracoabdominal ulcer. (A) Local skin blister appeared one week after cryoablation for chest wall metastasis; (B) Two months after procedure, a $4 \mathrm{~cm} \times 6 \mathrm{~cm}$ ulcer formed, with yellowish white necrosis at the base and black scab covering the surface; (C) Four months after procedure, the ulcer enlarged to $6 \mathrm{~cm} \times 8 \mathrm{~cm}$, and the surrounding skin showed obvious inflammatory erythema; (D) Six months after procedure, the ulcer further enlarged to $10 \mathrm{~cm} \times 10 \mathrm{~cm}$; (E) Nine months after procedure, also two months after PD- 1 antibody disuse, the ulcer shrank to $4 \mathrm{~cm} \times$ $7 \mathrm{~cm}$, most of the necrosis and black scab on the ulcer surface fell off, and fresh granulation tissue was visible around and at the base of the ulcer; ( $F$ ) Ten months after procedure, also three months after PD- 1 antibody disuse, the size of the ulcer was about $4 \mathrm{~cm} \times 7 \mathrm{~cm}$, the black scab completely fell off, the white necrosis decreased, and the fresh granulation tissue around as well as at the base of the ulcer increased.

\section{Supplementary Files}

This is a list of supplementary files associated with this preprint. Click to download.

- Tables1.docx 